\title{
Structural Analysis of the Glucocorticoid Receptor Ligand-Binding Domain in Complex with Triamcinolone Acetonide and a Fragment of the Atypical Coregulator, Small Heterodimer Partner
}

\author{
Emily R. Weikum, C. Denise Okafor, Emma H. D’Agostino, Jennifer K. Colucci, \\ and Eric A. Ortlund \\ Department of Biochemistry, Emory University School of Medicine, Atlanta, Georgia
}

Received February 1, 2017; accepted April 6, 2017

\section{ABSTRACT}

The synthetic glucocorticoids (GCs) dexamethasone, mometasone furoate, and triamcinolone acetonide are pharmaceutical mainstays to treat chronic inflammatory diseases. These drugs bind to the glucocorticoid receptor (GR), a ligand-activated transcription factor and member of the nuclear receptor superfamily. The GR is widely recognized as a therapeutic target for its ability to counter proinflammatory signaling. Despite the popularity of GCs in the clinic, long-term use leads to numerous side effects, driving the need for new and improved drugs with less off-target pharmacology. X-ray crystal structures have played an important role in the drug-design process, permitting the characterization of robust structure-function relationships. However, steroid receptor ligand-binding domains (LBDs) are inherently unstable, and their crystallization requires extensive mutagenesis to enhance expression and crystallization. Here, we use an ancestral variant of GR as a tool to generate a high-resolution crystal structure of GR in complex with the potent glucocorticoid triamcinolone acetonide (TA) and a fragment of the small heterodimer partner (SHP). Using structural analysis, molecular dynamics, and biochemistry, we show that TA increases intramolecular contacts within the LBD to drive affinity and enhance stability of the receptor-ligand complex. These data support the emerging theme that ligand-induced receptor conformational dynamics at the mouth of the pocket play a major role in steroid receptor activation. This work also represents the first GR structure in complex with SHP, which has been suggested to play a role in modulating hepatic GR function.

\section{Introduction}

The nuclear receptor superfamily is composed of a family of ligand-regulated transcription factors that are critical for maintaining specific gene-expression profiles across a number of biologic processes (Nagy and Schwabe, 2004). The glucocorticoid receptor (GR), the founding member of the nuclear receptor superfamily, is ubiquitously expressed and both upand down-regulates thousands of genes involved in immunity,

E.R.W is supported by a predoctoral NRSA awarded by the National Institutes of Health General Medical Sciences [Grant 1F31GM11339701A1]. E.A.O. is supported by the National Institutes of Health [Grant R01DK095750], American Heart Association [Grant 14GRNT20460124], and a W. M. Keck Foundation Medical Research Grant. C.D.O. is funded by a National Institutes of Health General Medical Sciences Institutional Research and Career Development Award [Grant K12 GM000680].

https://doi.org/10.1124/mol.117.108506. metabolism, and inflammation (Kadmiel and Cidlowski, 2013). GR has a modular architecture consisting of four primary domains: an N-terminal region that contains an activator function (AF-1) surface that interacts with coregulators; a highly conserved zinc finger DNA-binding domain; a flexible hinge region; and a ligand-binding domain (LBD), which binds ligands that modulate receptor activity. Ligands drive conformational changes within the LBD that modulate a second activation function surface (AF-2), which in turn enables selective interaction with coregulators (Carson-Jurica et al., 1990; Gronemeyer and Moras, 1995; Kumar and Thompson, 1999; Nagy and Schwabe, 2004). Unliganded GR resides in the cytosol bound to chaperone proteins. Upon binding to glucocorticoids, GR undergoes a conformational change, resulting in an exchange of chaperones and translocation to the nucleus (O'Malley and Tsai, 1992; Pratt and Toft, 1997).

ABBREVIATIONS: AF, activator function; CHAPS, 3-[(3-cholamidopropyl)dimethylammonio]-1-propanesulfonic acid; Dex, dexamethasone; GBS, glucocorticoid receptor binding sequences; GC, glucocorticoid; GR, glucocorticoid receptor; hGR, human GR; IPTG, isopropyl-thio-Dgalactopyranoside; LBD, ligand-binding domain; MBP, maltose-binding protein; MD, molecular dynamics; MF, mometasone furoate; NR, nuclear receptor; PDB, Protein Data Bank; RMSD, root mean square deviation; SHP, small heterodimer partner; SR, steroid receptor; TA, triamcinolone acetonide; TIF2, nuclear receptor coactivator-2. 
In the nucleus, GR interacts with the genome via multiple mechanisms to then recruit transcriptional coregulator proteins, which serve to promote or repress transcription (Darimont et al., 1998; Hu and Lazar, 1999). The simplistic view of GR signaling suggests that GR will bind directly to DNA at canonical GR binding sequences (GBS) as a homodimer to activate transcription or can bind as a monomer to inverted repeat GBS (IR-GBSs/negative glucocorticoid response elements) to repress transcription (Luisi et al., 1991; Hudson et al., 2013). GR can also regulate transcription in a DNA-independent manner by interacting directly with the proinflammatory transcription factors activator protein-1 and nuclear factor $\kappa \mathrm{B}$ through protein-protein interactions to block their activity (Mordacq and Linzer, 1989; Schüle et al., 1990; Yang-Yen et al., 1990). This mechanism, known as tethering, is the major mechanism targeted by pharmaceutical companies for anti-inflammatory therapies (De Bosscher et al., 2000).

Since their discovery in 1948, glucocorticoids (GCs) have been the most efficacious treatment of chronic inflammatory diseases, such as rheumatoid arthritis and asthma (Cato and Wade, 1996). GC-bound GR is unmatched in its antiinflammatory action, resulting in its prevalence in the clinic and worldwide sales that surpass $\$ 10$ billion per year (Clark and Belvisi, 2012). Yet despite the effectiveness of GCs, longterm exposure leads to numerous debilitating side effects, including weight gain, muscle wasting, and development of Cushing's syndrome (Schäcke et al., 2002). These adverse effects have generally been attributed to GR's role in transactivation (Schäcke et al., 2002), spurring studies focused on the molecular mechanisms of GR-ligand interactions to support the development of more selective GCs.

Triamcinolone acetonide (TA) is a highly potent synthetic glucocorticoid that has been approved by the Food and Drug Administration to treat allergic rhinitis as a nasal spray (Nasacort; Sanofi, Bridgewater, NJ) (Doggrell, 2001; Fredman and Tenenhaus, 2013). It is also used to treat macular edema as a result of diabetes (KENALOG) and as an over-thecounter cream to treat skin lesions (Bristol-Myers Squibb, Princeton, NJ) (Fredman and Tenenhaus, 2013). In addition to its prolific clinical uses, TA has been widely used in the laboratory setting to probe GR biology since it shows a higher affinity than dexamethasone (Dex) and promotes the soluble expression and purification of the full-length receptor for in vitro biochemical characterization (Derendorf and Meltzer, 2008; Nehmé et al., 2009; Bain et al., 2012; Robblee et al., 2012).

Soluble expression and purification of human GR LBD is challenging and requires mutation at several sites to promote solubility and purification (Gebhardt et al., 2013). These mutations, designed via sequence alignments to other steroid receptor LBDs, enable expression in Escherichia coli and greatly enhance crystallization. However, their effect on ligand selectivity, conformational dynamics, and activity remains unknown, resulting in uncertainty when constructing robust structure-function relationships. Recently, resurrected ancestral proteins have been used as a tool not only to study evolutionary biology, but also to generate X-ray crystal structures of novel complexes (Bridgham et al., 2009; Kohn et al., 2012; Colucci and Ortlund, 2013). Here, we use AncGR2, the ancestral precursor to the modern GR, which recapitulates both the structure and function of its extant counterpart while exhibiting better expression, purification, and crystallization in the laboratory setting along with a higher tolerance to mutagenic analysis, which is critical for probing structurefunction relationships (Ortlund et al., 2007; Bridgham et al., 2009; Thiruchelvam et al., 2011; Kohn et al., 2012). AncGR2 and human GR (hGR) LBDs share $78.6 \%$ sequence identity with $100 \%$ of the residues within the ligand-binding pocket conserved. This makes AncGR2 an ideal surrogate for hGR structure-function studies, and allowed us to generate the first X-ray crystal structure of the GR LBD complexed with TA and with a peptide from the atypical coregulator, small heterodimer partner (SHP).

\section{Materials and Methods}

Protein Expression and Purification. AncGR2 LBD (GenBank accession number EF631976.1) was cloned into a pMALCH10T vector, transformed into $E$. coli strain BL21(pLysS), and expressed as a maltose-binding protein (MBP) with a hexahistidine tag. Cultures (9 l in Terrific Broth (12 g/L Tryptone, $24 \mathrm{~g} / \mathrm{L}$ yeast extract, $4 \mathrm{~mL}$ glycerol, $2.3 \mathrm{~g} / \mathrm{L} \mathrm{KH} 2 \mathrm{PO} 4,12.5 \mathrm{~g} / \mathrm{L} \mathrm{K} 2 \mathrm{HPO} 4)$ ) were grown at $37^{\circ} \mathrm{C}$ until an the sample reaches an optical density of 0.6 at a wavelength of $600 \mathrm{~nm}$. Protein expression was induced with the addition of $0.5 \mathrm{mM}$ isopropyl-1-thio-D-galactopyranoside (IPTG) and $25 \mu \mathrm{M}$ TA or $50 \mu \mathrm{M}$ dexamethasone and grown for 4 hours at $32^{\circ} \mathrm{C}$. Cells were lysed in a buffer containing $20 \mathrm{mM}$ Tris-HCl (pH 7.4), $300 \mathrm{mM} \mathrm{NaCl}, 25 \mathrm{mM}$ imidazole, and $5 \%$ glycerol via sonication on ice. Fusion protein was purified by affinity chromatography (HisTrap FF; GE Healthcare, Little Chalfront, UK), and the MBP tag was removed by tobacco etch virus protease cleavage with an additional two passes over HisTrap FF medium (GE Healthcare). After purification, protein was dialyzed overnight at $4^{\circ} \mathrm{C}$ into buffer containing $20 \mathrm{mM}$ HEPES (pH 8.0), $200 \mathrm{mM} \mathrm{NaCl}, 10 \%$ glycerol, and $50 \mu \mathrm{M} 3-[(3-$ cholamidopropyl)dimethylammonio]-1-propanesulfonic acid (CHAPS). Protein was concentrated to $3-5 \mathrm{mg} / \mathrm{ml}$, flash frozen in liquid $\mathrm{N}_{2}$, and stored at $-80^{\circ} \mathrm{C}$.

Protein Crystallization, Data Collection, and Structure Determination. AncGR2 LBD-TA was concentrated to $4.75 \mathrm{mg} / \mathrm{ml}$

\section{TABLE 1}

Data collection and refinement statistics

Data were collected from a single crystal; values in parentheses are for the highestresolution shell.

\begin{tabular}{lc}
\hline & AncGR2 LBD-TA + SHP \\
\hline Data collection & \\
Space group & $\mathrm{C} 2$ \\
Cell dimension & $\mathrm{a}=87.0, \mathrm{~b}=52.8, \mathrm{c}=126.0$ \\
Resolution $(\AA)$ & $2.11(2.19-2.11)$ \\
$\mathrm{R}_{\text {pim }}$ & $6.2(37.0)$ \\
$\mathrm{I} / \sigma$ & 2.67 \\
Completeness & $98.6(93.2)$ \\
Redundancy & $3.6(3.0)$ \\
Refinement & \\
Resolution & 2.11 \\
No. reflections & 31,942 \\
$\mathrm{R}_{\text {work }} / \mathrm{R}_{\text {free }}$ & $21.5 / 25.2$ \\
No. atoms & \\
Protein & 4158 \\
Ligands & 62 \\
Water & 119 \\
$B$-factors & \\
Protein & 44.9 \\
Ligands & 31.8 \\
Water & 41.0 \\
RMSD & \\
Bond lengths $(\AA)$ & 0.002 \\
Bond angles $\left({ }^{\circ}\right)$ & 0.52 \\
PDB Code & $5 \mathrm{UFS}$ \\
\hline
\end{tabular}


and incubated with a peptide derived from SHP (NH2QGASRPAILYALLSSSLK-OH) at 2-fold molar excess. Crystals were grown by hanging drop vapor diffusion at $18^{\circ} \mathrm{C}$ in drops containing $1 \mu \mathrm{l}$ of AncGR2-TA-SHP and $1 \mu \mathrm{l}$ of $100 \mathrm{mM}$ HEPES (pH 7.5), 1.5\% glycerol, and $25 \%$ polyethylene glycol 300 . Crystals were flash frozen in liquid nitrogen using a cryopreservative consisting of $100 \mathrm{mM}$ HEPES (pH 7.5), 10\% glycerol, and 40\% polyethylene glycol 300 . Data were collected remotely from the Southeast Regional Collaborative Access Team at the Advanced Photon Source, 22ID beamline (Argonne National Laboratories, Chicago, IL). Data were processed and scaled using HKL-2000 (HKL Research, Inc., Charlottesville, VA) (Otwinowski and Minor, 1997) and phased by molecular replacement using Phaser-MR (Phenix, Berkeley, CA) (Adams et al., 2010). The structure was phased using the previously solved AncGR2dexamethasone-nuclear receptor coactivator-2 (TIF2) peptide complex as a search model (3GN8) (Bridgham et al., 2009). Structure refinement and validation was performed using PHENIX (Phenix, Berkeley, CA) (version 1.11.1), and model building was performed in COOT (MRC Laboratory of Molecular Biology, Cambridge, UK) (Adams et al., 2010; Emsley et al., 2010). PDB Redo was used iteratively to optimize refinement parameters and geometry (Joosten et al., 2009). PyMOL (version 1.8.2; Schrödinger, New York, NY) was used to visualize structures and generate figures.

$R_{\text {factors }}$ for the final model are 21.5 and $25.2 \%$ for $R_{\text {work }}$ and $R_{\text {free, }}$, respectively. MolProbity (Duke University, Durham, NC) was used for model validation, indicating that $98 \%$ of the residues fall in the most favored regions of the Ramachandran plot with none in disallowed regions (Davis et al., 2007; Adams et al., 2010). The overall MolProbity score was 0.70 , placing the structure in the 100th percentile for overall geometric quality among protein crystal structures of comparable resolution. The remaining data collection and refinement statistics can be found in Table 1. Coordinates and structure factors were deposited in the Protein Data Bank (PDB) with the accession number 5UFS.

Ligand Binding and Competition Assays. Hexahistidinetagged MBP-fused AncGR2 LBD-Dex was dialyzed overnight into buffer containing $150 \mathrm{mM} \mathrm{NaCl}, 10 \mathrm{mM}$ HEPES (pH 7.4), 3 mM EDTA, $5 \mathrm{mM}$ dithiothreitol, and $0.005 \%$ Tween 20 . All binding experiments were performed using this buffer. Binding affinity for dexamethasonefluorescein was measured with $12 \mathrm{nM}$ dexamethasone-fluorescein and protein concentrations from $10^{-10}$ to $10^{-5} \mathrm{M}$. Polarization was monitored on a Neo plate reader (Biotek, Winooski, VT) at an excitation/ emission wavelength of $485 / 528 \mathrm{~nm}$. Three technical replicates and three biologic replicates were conducted, and graphs are a compilation of all data collected. Binding data were fit with a one-site binding curve in GraphPad Prism version 7 (GraphPad, Inc., La Jolla, CA). Competition assays were performed at a protein concentration 1.2 times the binding affinity for dexamethasone and in the presence of $12 \mathrm{nM}$ dexamethasone-fluorescein and $10^{-10}$ to $10^{-5} \mathrm{M}$ of competing ligand. Three technical replicates and three biologic replicates were conducted, and graphs are a compilation of all data collected. GraphPad Prism version 7 was used to analyze data using a one-site, fit $K_{i}$ curve.

Differential Scanning Fluorimetry. Differential scanning fluorimetry assays for protein-ligand complexes (Fig. 3D) were performed with $5 \mu \mathrm{M}$ of AncGR2 LBD (expressed and purified with varying ligands); buffer containing $20 \mathrm{mM}$ Tris (pH 7.4), $300 \mathrm{mM} \mathrm{NaCl}$, and $5 \%$ glycerol; and a final 1:1000 dilution of SYPRO orange dye (Sigma-Aldrich, St. Louis, MO) to a final volume of $20 \mu \mathrm{l}$. Reactions with coregulator peptides (Fig. $5 \mathrm{E}$ ) consisted of $5 \mu \mathrm{M}$ protein, $5 \mu \mathrm{M}$ peptide, buffer, and SYPRO dye, as described earlier. The peptide sequences used were as follows: SHP nuclear receptor (NR) box 1 (NH2-QGASRPAILYALLSSSLK-OH) and TIF2 NR box 3 (NH2KENALLRYLLDKDD-OH). For all differential scanning fluorimetry experiments, reactions were heated from $25^{\circ} \mathrm{C}$ to $95^{\circ} \mathrm{C}$ in $0.5^{\circ} \mathrm{C}$ increments every minute, and fluorescence was monitored using the ROX filter $(602 \mathrm{~nm})$ with a StepOnePlus Real Time PCR System (Thermo Fisher (Waltham, MA)). Two technical replicates and three biologic replicates were conducted, and graphs are a compilation of all data collected. Data were normalized by subtracting each data point from a dye-buffer only control reaction. Data were fit using a Boltzmann sigmoidal curve to determine the melting temperature $\left(\mathrm{T}_{\mathrm{m}}\right)$, defined as $50 \%$ unfolding.

ProSMART Analysis. The ProSMART (MRC Laboratory of Molecular Biology, Cambridge, UK) alignment tool provides a conformationindependent structural comparison of two proteins (Nicholls et al., 2014). Pairwise comparisons were conducted between AncGR2 LBD-TA-SHP and AncGR2 LBD-Dex-TIF2 (PDB 3GN8); AncGR2 LBD-TA-SHP and AncGR2 LBD-mometasone furoate (MF)-TIF2 (PDB 4E2J); and AncGR2 LBD-Dex-TIF2 and AncGR2 LBD-MF-TIF2 (Bridgham et al., 2009; Kohn et al., 2012). The comparisons generate a Procrustes score, which is mapped onto the LBD structures. This score is the root mean square deviation (RMSD) of the central residue of two corresponding structural fragments of length $n$, where $n$ is an odd number of amino acids.

Molecular Dynamics Simulations. Three complexes were prepared for molecular dynamics simulations using AncGR2 LBD: 1) TA-TIF2, 2) Dex-TIF2 (PDB 3GN8), and 3) MF-TIF2 (PDB 4E2J). The structure for TA-TIF2 was created by replacing the SHP peptide with TIF2 and an all-atom minimization of the structure. The complexes were solvated in an octahedral box of transferable intermolecular potential 3P (Jorgensen et al., 1983) water with a 10-Å buffer around the protein complex. $\mathrm{Na}^{+}$and $\mathrm{Cl}^{-}$ions were added to neutralize the protein and achieve physiologic conditions to a final concentration of $150 \mathrm{mM}$. All systems were set up using xleap in AmberTools (AMBER, University of California, San Francisco, CA) (Case and Kollman, 2012) with the parm99-bsc0 forcefield (Pérez et al., 2007). Parameters for TA, MF, and Dex were obtained using Antechamber (Case et al., 2014) in AmberTools. All minimizations and simulations were performed with Amber14 (Case et al., 2014). Systems were minimized with 5000 steps of steepest decent followed by 5000 steps of conjugate gradient minimization with $500-\mathrm{kcal} / \mathrm{mol} \bullet \AA^{2}$ restraints on all atoms. Restraints were removed from all atoms, excluding the atoms in both the ligand and the TIF2 peptide, and the previous minimization was repeated. The systems were heated from 0 to $300 \mathrm{~K}$ using a 100-ps run with constant volume periodic boundaries and $5-\mathrm{kcal} / \mathrm{mol} \bullet \AA^{2}$ restraints on all protein and ligand atoms. Twelve nanoseconds of molecular dynamics (MD) equilibration was performed with $10-\mathrm{kcal} / \mathrm{mol} \cdot \AA^{2}$ restraints on protein and ligand atoms using the NPT (constant number of particles, pressure, and temperature) ensemble. Restraints were reduced to $1 \mathrm{kcal} / \mathrm{mol} \cdot \AA^{2}$ for an additional $10 \mathrm{~ns}$ of MD equilibration. Then restraints were removed, and 500-ns production simulations were performed for each system in the NPT ensemble. A 2 -fs time step was used, and all bonds between heavy atoms and hydrogens were fixed with the SHAKE algorithm (Ryckaert et al., 1977). A cut-off distance of $10 \AA$ was used to evaluate long-range electrostatics with particle mesh ewald and for van der Waals forces. Twenty-five thousand evenly spaced frames were taken from each simulation for analysis.

MD trajectories were analyzed with various tools. Structural averaging and analysis were performed with the CPPTRAJ module (Roe and Cheatham, 2013) of AmberTools (version 14). Hydrogen bonds were identified using HBPLUS (EMBL-EBI, Cambridgeshire, UK) (McDonald and Thornton, 1994) with default criteria (minimum angles: D-H-A 90.0, H-A-AA 90.0, D-A-AA 90; maximum distances: D-A 3.9A, H-A 2.5 $; \mathrm{D}=$ donor, $\mathrm{A}=$ acceptor, $\mathrm{AA}=$ atom attached to acceptor). Root mean square fluctuation analysis was performed on $\mathrm{C} \alpha$ atoms of protein residues. Root mean square fluctuation was computed relative to the reference structure (crystal structure) for each frame in the trajectory. CPPTRAJ was used to calculate distances between $\mathrm{C} \alpha$ atoms or hydrogen-bonding atoms over trajectories. All distances between residue pairs were calculated using $\mathrm{C} \alpha$ atoms. The MMTSB toolset (The Scripps Research Institute, Jupiter, FL) was used to perform a cluster analysis with a 2-Å RMSD cutoff (Feig et al., 2004).

\section{Results}

Structural Analysis of AncGR2-Triamcinolone/SHP Complex. To understand how TA interacts with GR, we determined the X-ray crystal structure of the AncGR2 LBD-TA 
A

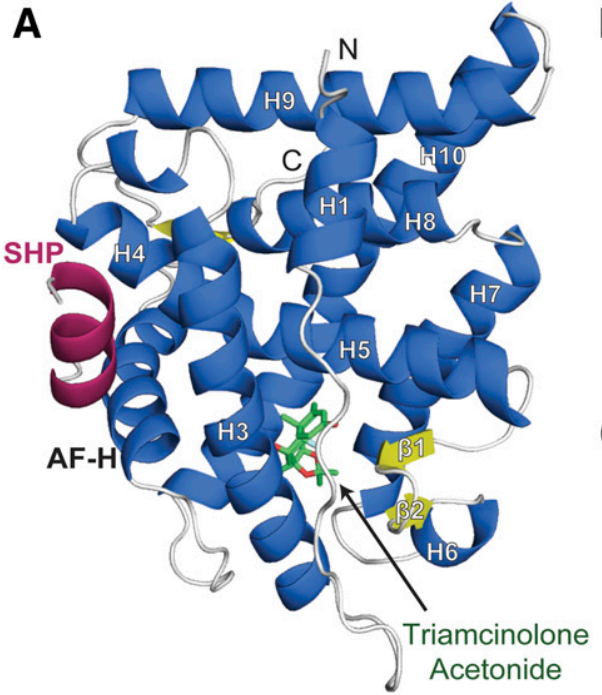

B

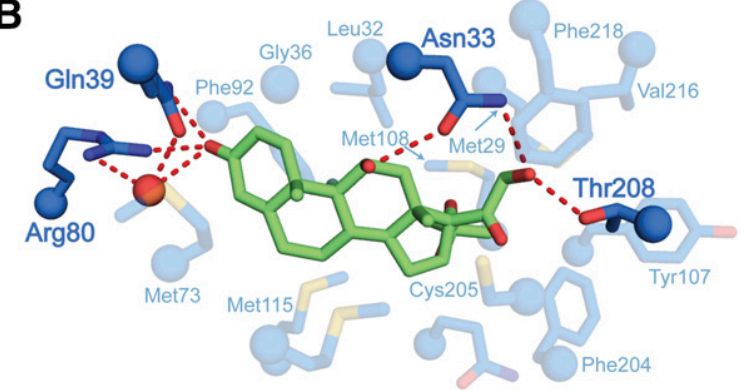

C
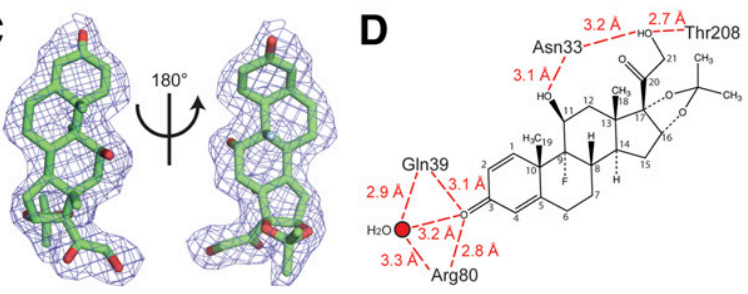

Fig. 1. Structure of the AncGR2-TA-SHP NR box 1 complex. (a) Illustration representing the overall structure of AncGR2 LBD (blue) in complex with TA (green) and SHP (pink). (b) Close-up view of ligand-binding pocket (side chains are shown as sticks with $\alpha$-carbons shown as spheres). Residues that participate in hydrophobic contacts are shown in faded blue, and side chains that make hydrogen-bonding interactions are shown in darker blue with hydrogen bonds denoted by red dashes. (c) Omit map $\left(\mathrm{F}_{\mathrm{o}}-\mathrm{F}_{\mathrm{c}}\right)$ contoured at $2.5 \sigma$ around the ligand. (d) Schematic of hydrogen-bonding network. Hydrogen bonds are shown in red.

complex bound to a fragment of the coregulator SHP. Crystals formed in the C2 space group with two AncGR2 LBD-TA-SHP complexes in the asymmetric unit. Data sets were collected to $2.1 \AA$ with $98.6 \%$ completeness. Full data collection and refinement statistics are summarized in Table 1.

The AncGR2 LBD is bound by one molecule of TA and the SHP coregulator peptide (Fig. 1A). The LBD adopts a canonical fold with $11 \alpha$-helices and four $\beta$-strands that fold into three layers of a helical sandwich bundle (Fig. 1A) (Kumar and Thompson, 1999). This folding creates a hydrophobic ligandbinding pocket encompassing TA (Fig. 1B), which is supported by unambiguous electron density (Fig. 1C).

TA is coordinated via extensive hydrophobic contacts and a series of specific hydrogen-bonding interactions (Fig. 1D). This hydrogen-bonding network includes a water molecule, which is a common feature for steroid receptor (SR) LBD-ligand complexes and is seen in all AncGR2 LBD-ligand structures to date (Bridgham et al., 2009; Kohn et al., 2012). This water hydrogen bonds with the terminal oxygen of the A-ring. The amide of Gln39 and guanidinium group of Arg80 not only hydrogen bond to the water molecule but also form hydrogen bonds to the A-ring 3-keto oxygen (Figs. 1D and 2A). MD simulations of ligand-hydrogen-bonding interactions show that, over a 500-ns simulation, these hydrogen bonds alternate between being direct or water-mediated (Fig. 2A). In addition, there is a water-mediated hydrogen bond that occurs between the Arg80 and Gln39 residues that persists for $47 \%$ of the simulation. Both of these hydrogen-bonding interactions have a joint effect in keeping TA in the correct orientation within the ligand-binding pocket over the duration of the simulation. Asn33 hydrogen bonds to the C-ring 11-hydroxyl. Finally, Asn 33 and Thr208 hydrogen bond to the 21-hydroxyl located off the C-17 position on the D-ring. These hydrogen
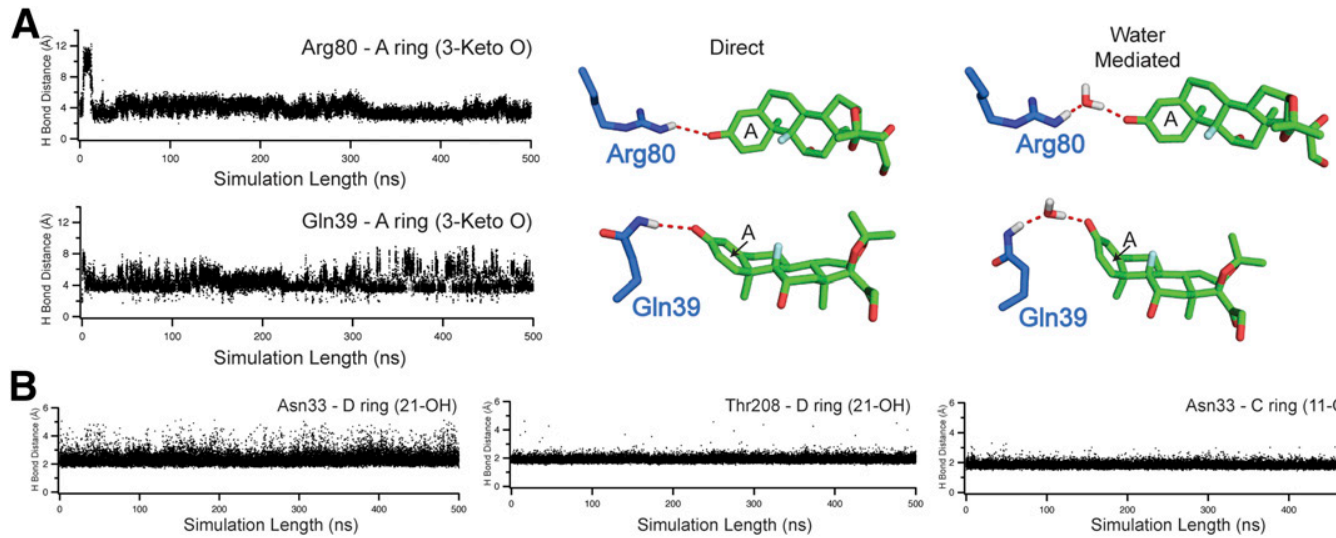

B
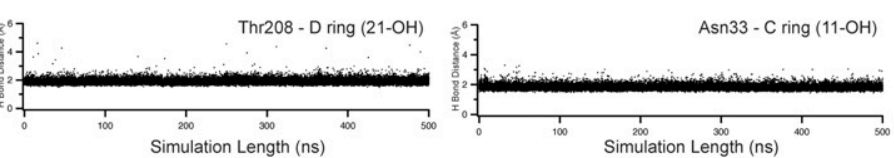

Fig. 2. MD simulations support ligand-binding pocket-hydrogen-bonding interactions. (a) Graph of hydrogen-bonding distances between the guanidinium hydrogen atom on Arg80 and the amide hydrogen atom on Gln39 with the 3-keto oxygen on the A-ring of TA during the 500-ns MD simulation. These hydrogen atoms either make contact with the water molecule within the pocket or make direct interactions with the ligand. The A-ring of TA is labeled in each view of the ligand. (b) MD analysis supports the hydrogen-bonding interactions seen between Asn33 and Thr308 with the ligand. 


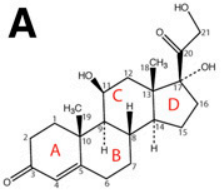

Cortisol

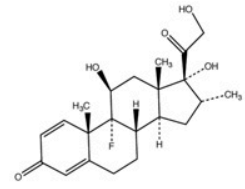

Dexamethasone

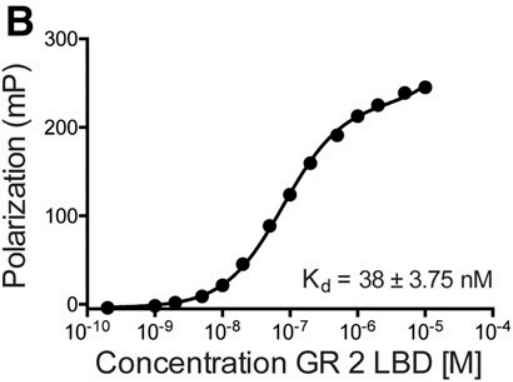

D

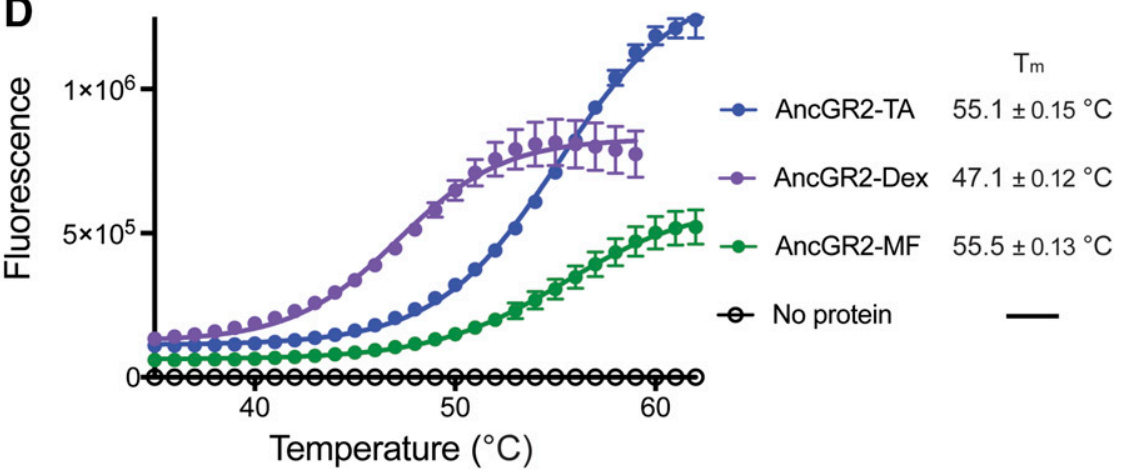

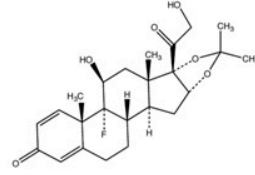

Triamcinolone Acetonide

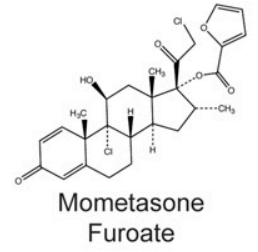

C

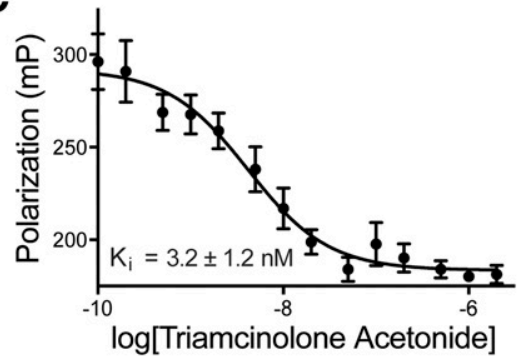

Fig. 3. TA readily competes Dex out of the binding pocket and highly stabilizes the LBD. (a) GR's endogenous ligand cortisol and a myriad of synthetic glucocorticoids, including Dex, TA, and MF. (b) Fluorescent Dex binds AncGR2 LBD with a $\mathrm{K}_{\mathrm{d}}$ of $38 \mathrm{nM}$. Binding was measured via fluorescence polarization, and graphs are fit using a one-site binding curve to calculate $\mathrm{K}_{\mathrm{d}}$ values; error bars indicate standard error of the mean (S.E.M.; $n=9$ ). (c) TA competes fluorescent Dex out of the ligand-binding pocket with a $\mathrm{K}_{\mathrm{i}}$ of $3.2 \mathrm{nM}$. Graphs are fit using a one-site, fit $\mathrm{K}_{\mathrm{i}}$ equation to calculate $\mathrm{K}_{\mathrm{i}}$ values; error bars indicate S.E.M. $(n=9)$. (d) Differential scanning fluorimetry monitors the thermal stability of different AncGR2 ligand complexes. Both TA and MF-bound LBD increased the thermal stability by $8^{\circ} \mathrm{C}$. Graphs are fit using the Boltzmann sigmoidal equation, which calculates $\mathrm{T}_{\mathrm{m}}$ as $50 \%$ unfolding. Error bars error bars indicate S.E.M. $(n=6)$. bonds are well supported via $\mathrm{MD}$ and persist throughout the entirety of the simulations (Fig. 2B).

The C-17 Acetonide Moiety on TA Increases the Affinity and Stability for GR LBD over Dex. Synthetic GCs typically show increased affinity and selectivity for the receptor over the GR's endogenous ligand, cortisol. Corticosteroids, similar to most cholesterol-derived steroid hormones, contain three 6-carbon rings (A, B, C) and one 5-carbon ring (d) (Fig. 3A). Dex varies from cortisol by the addition of a C1-C2 double bond in ring $\mathrm{A}$, a C-16 $\alpha$-methyl, and a C-9 $\alpha$-fluoro group. TA varies from Dex by the addition of the 16,17 acetonide moiety. MF varies from Dex by the presence of a chloro group at C-9 instead of a fluoro group, a chloro group instead of a 21-hydroxyl off the C-17 D-ring, and the large furoate moiety off the $\mathrm{C}-17$ position. The $\mathrm{C}-17$ furoate moiety is more polar and labile than the TA acetonide moiety and is cleaved in vivo. The active metabolite, mometasone, contains a hydroxyl at the C-17 position due to loss of the furoate moiety; thus, conclusions based on analysis of the GR-MF complex cannot be reliably correlated with its in vivo potency. However, MF has proven a useful tool to probe GR LBD biochemistry in vitro and is therefore included in our analysis. For synthetic GCs, the steroid backbone halogen substitutions are thought to increase potency, and the substitutions at position 17 account for the largest functional differences (Bikowski et al., 2006; Derendorf and Meltzer, 2008). These substitutions on TA and MF drive the increased affinity and reported potencies over Dex (Grossmann et al., 2004; Nehmé et al., 2009).

Similar to previous reports, we show that TA displaces bound fluorescent Dex from the AncGR2 LBD with a $K_{i}$ of $3.2 \mathrm{nM}\left(\mathrm{K}_{\mathrm{d}}\right.$ for fluorescent Dex $=38 \mathrm{nM}$ ) (Fig. 3B) (Yoneda et al., 1995; Bledsoe et al., 2002; Grossmann et al., 2004; Nehmé et al., 2009). Furthermore, we show there is an $8^{\circ} \mathrm{C}$ increase in thermal stability of the LBD when bound by TA or MF over Dex (Fig. 3C). To understand how TA has such a high affinity and potency for GR, we compared our structure to the previously solved AncGR2-Dex-TIF2 and AncGR2-MF-TIF2 complexes (PDB 3GN8, 4E2J) (Bridgham et al., 2009; Kohn et al., 2012) (Fig. 4).

Superposition of the different ligand-bound structures revealed only subtle differences in the RMSDs of main chain carbon alphas. The difference between AncGR2-TA and AncGR2-Dex is $0.346 \AA$, and between AncGR2-TA and AncGR2-MF is $0.349 \AA$. Within the ligand-binding pocket, the same side chains participate in hydrogen-bonding interactions, including a water molecule in all structures (Fig. 4A). This is not surprising when comparing Dex and TA, as the ligands are almost identical except for the acetonide moiety, which is contacted by hydrophobic interactions. However, MF-bound GR makes one fewer hydrogen-bonding interaction; the C-17 position contains a chloro group rather than a hydroxyl, altering the interaction with Thr208. Yet, as with $\mathrm{MF}$, the acetonide $\mathrm{C}-17$ addition is oriented almost $90^{\circ}$ from the steroid backbone. This allows for the ligand to expand and fill the binding pocket as well as generate additional hydrophobic contacts between GR and the ligand. These large substitutions on TA and MF cause a $100-\AA^{3}$ (1.1-fold) and $200-\AA^{3}$ (1.3-fold) increase, respectively, in pocket volume compared with Dex (Fig. 4B). Therefore, the differences in potency and affinity must be due to changes in hydrophobic contacts and long-range allosteric changes generated by the 

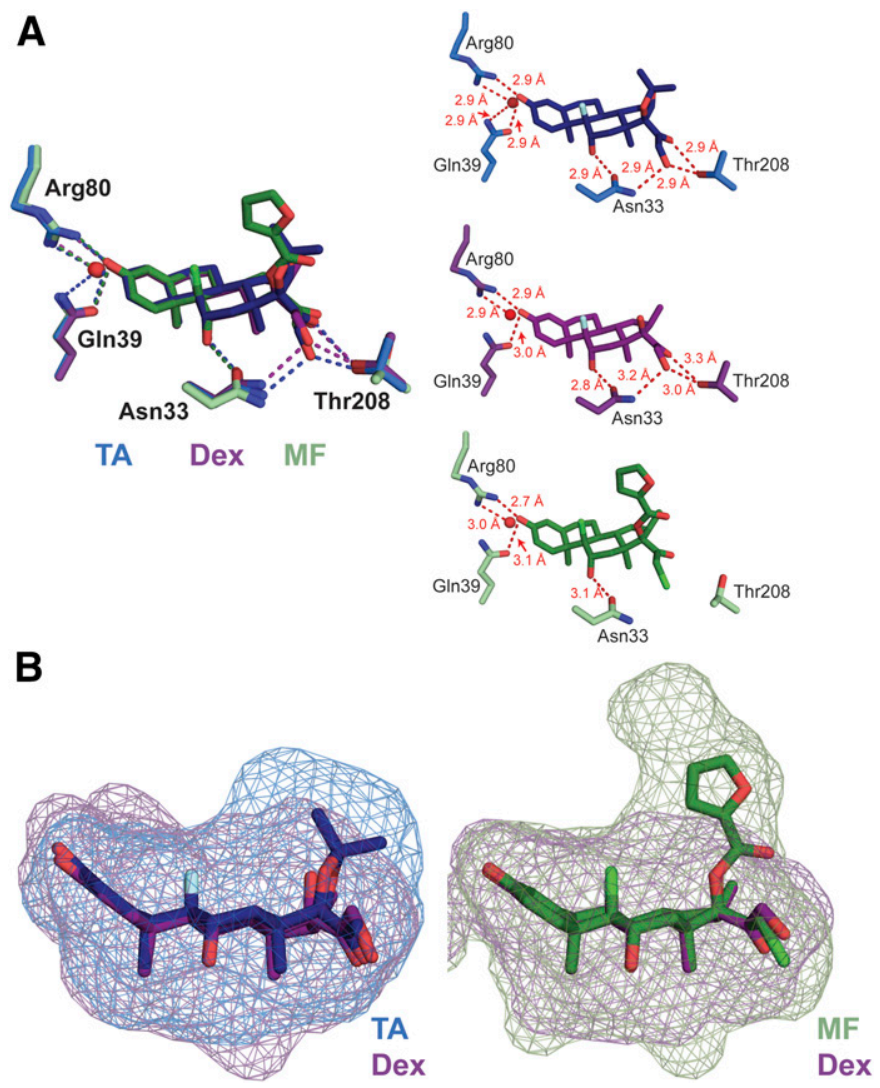

Fig. 4. Comparison of glucocorticoid ligands bound to AncGR2 LBD. (a) Overlay of TA (blue), Dex (purple), and MF (green) within the pocket. Insets to the right show TA (blue), Dex (purple), and MF (green) hydrogenbonding networks. Hydrogen bonds are shown in red. (b) Mesh depicting ligand-pocket volume induced by different glucocorticoid ligands. TA and MF have large additions at the C-17 position, which the GR ligand-binding pocket expands to accommodate.

ligands. To investigate this possibility, we combined in-depth structural analysis with MD simulations.

TA Increases Intramolecular Contacts within the LBD to Drive Affinity and Stability. To identify how differential ligands affect local structure, we performed pairwise comparisons of the three AncGR2 ligand structures using ProSMART (Nicholls et al., 2014) (Fig. 5A). Each chain is compared separately, and the final models are colored by their Procrustes score, which defines the similarity of an aligned fragment according to the legend provided in Fig. 4. The largest differences are seen in the mouth of the ligand-binding pocket, encompassing the loops after $\mathrm{H} 1, \mathrm{H} 3$, and $\mathrm{H} 6$, and the transition between H9 and H10. Differences in the H9-H10 loop are not surprising, as this loop is often difficult to model and is not supported by strong electron density in any structure. However, there are meaningful differences in local residue conformation at the entrance to the ligand-binding pocket, where TA constricts the opening of the pocket to $4.6 \AA$, compared with $6.3 \AA$ for Dex and $10.1 \AA$ for MF as measured by the distance between Ser25 on H3 and Pro106 on H6 (Fig. 5B). This TA-driven pocket constriction is also observed in MD simulations. The average Ser25-Pro106 distances in the simulations are 6.4, 10.5, and $11.3 \AA$ for TA, Dex, and MF, respectively. To link differential contacts with ligand-induced differences in receptor motion, we used MD to calculate the root mean square fluctuations between the key LBD-ligand complexes over 500-ns simulations. To focus only on ligandinduced dynamic changes, we first replaced SHP with TIF2 to mitigate peptide-driven effects. The MD trajectories showed less motion at residues 101-109 (helix 6-7) in the TA complex compared with both Dex and MF, suggesting TA introduces a stabilizing effect (Fig. 5, C and D).

To obtain the structure most representative of the dominant conformation sampled in each trajectory, clustering was performed with the MMTSB tool. For each AncGR2 complex, the structure with the lowest RMSD in the most populated cluster was used as a representative for the complex. Comparison of these structures shows a rearrangement of the residues in the binding pocket of the TA-bound structure as a result of stabilizing, hydrophobic interactions between the acetonide group and Met29 and Met108 (Fig. 5E). The interaction causes a shift of other residues (e.g., Met103) and shifts in the overall positions of $\mathrm{H} 6$ and $\mathrm{H} 7$, bringing them closer (Fig. 5, F and G). This movement is responsible for the closer distances observed between Ser25 and Pro106 in the TA-bound structure. Similar effects are observed in AncGR2 LBD-TA-SHP and LBD-TA-TIF2 structures, indicating that the TA ligand drives this effect.

Recognition of the SHP NR Box 1 LXXLL Motif. Coregulator proteins interact with SRs via an $\alpha$-helix containing a short LXXLL motif ( $\mathrm{L}$, leucine; $\mathrm{X}$, any amino acid) (Jenkins et al., 2001; Millard et al., 2013). These coregulators bind to the same hydrophobic groove on the surface of SR LBDs, the AF-2 (Heery et al., 1997; Vandevyver et al., 2014). The AF-2 is composed of helices 3, 4, and 12 and is generally held in place by a charge clamp formed by a lysine on $\mathrm{H} 3$ and a glutamate on H12 that interacts with the helix dipole (Bledsoe et al., 2002; Vandevyver et al., 2014). GR is known to interact with a wide variety of coregulator proteins, yet structural analysis has been limited to the typical coregulators of the p160 SRC family, specifically SRC-2 (TIF2, GRIP-1). Here, we show GR complexed with 11 residues of the SHP NR box 1 peptide. This is the first GR LBD structure with this coregulator.

SHP is an atypical orphan nuclear receptor composed of an LBD only that cofolds with the transcriptional corepressor EID1 (Seol et al., 1996; Lee et al., 2007; Zhi et al., 2014). SHP has been shown to act as a potent corepressor for numerous nuclear receptors, including estrogen receptor, retoinoid $\mathrm{X}$ receptor, LRH-1, and GR (Johansson et al., 1999, 2000; Lee et al., 2000; Borgius et al., 2002; Ortlund et al., 2005). To modulate receptor activity, SHP utilizes two canonical NR box motifs to bind to the AF-2 surface, competing directly with coactivators (Lee et al., 2000). SHP repression of GR activity has been postulated to have important biologic implications in the liver and pancreas (Borgius et al., 2002).

The overall AncGR2-TA-SHP structure shows the AF-H in the active conformation and the SHP peptide bound at the $\mathrm{AF}$ surface (Fig. 6A). The peptide inserts leucine side chains into the surface and is further stabilized by a charge clamp interaction, which is conserved across NRs. Lys48 on H3 hydrogen bonds with the backbone carbonyl of Leu24, and Glu224 hydrogen bonds to the free amide nitrogen of Ile20 (Fig. 6B). The terminus of the peptide is stabilized by a hydrogen bond involving Lys48 interacting directly with the terminal Ser27 residue. The peptide is further stabilized by Met62, which makes van der Waals contacts with Tyr22. As in previous NR LBD-SHP structures, this interaction holds the tyrosine residue in the center of the 


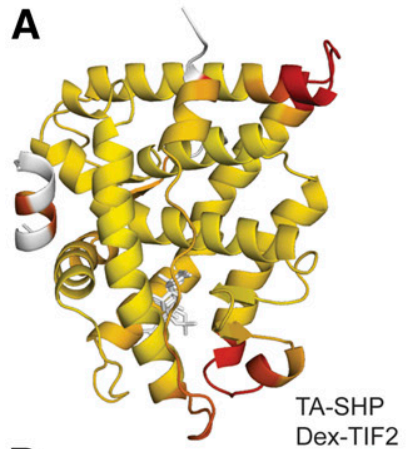

B

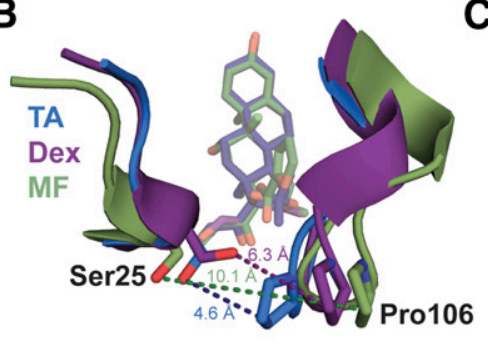

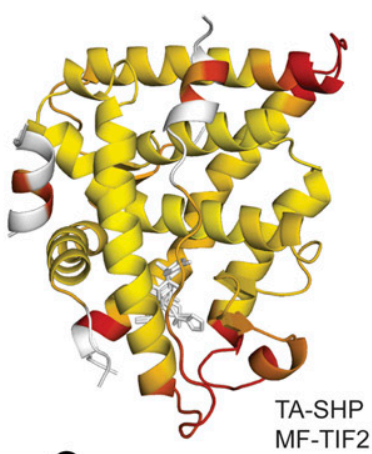
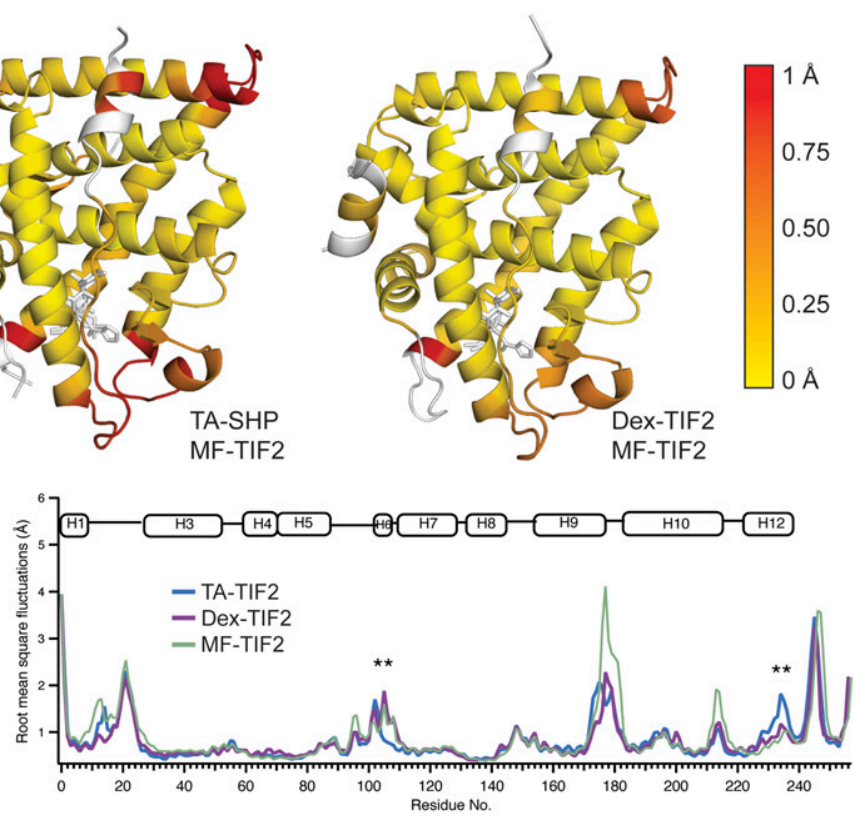

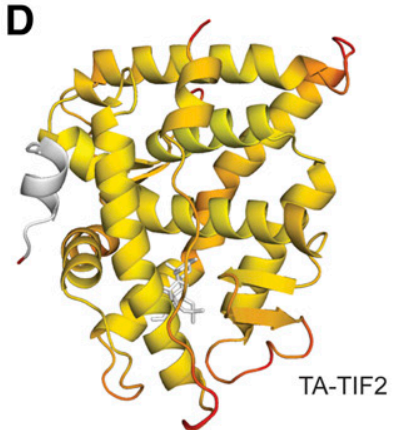

E

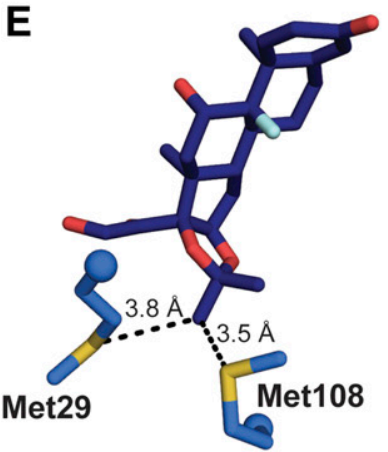

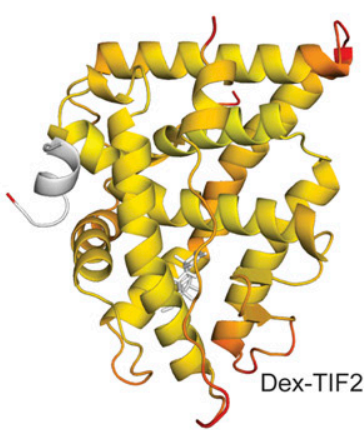

$\mathbf{F}$

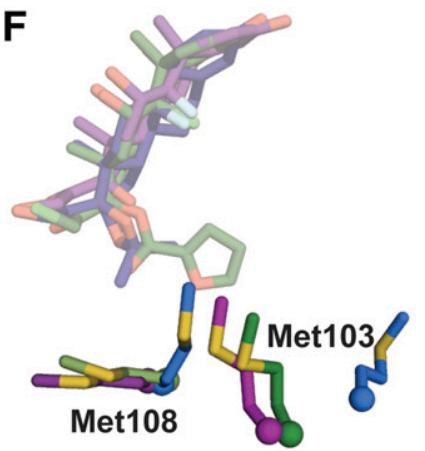

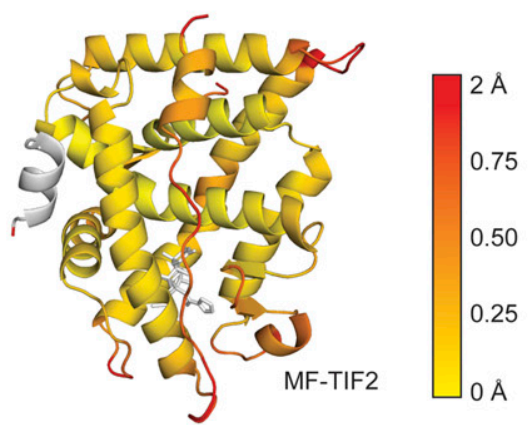

G

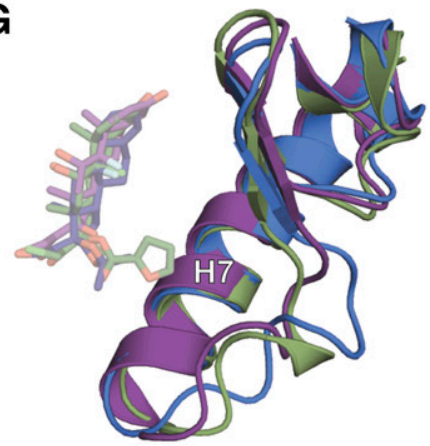

Fig. 5. Structural comparison of different GR-ligand complexes. (a) ProSMART analysis of pairwise comparisons of different structures of GR-ligand complexes. Areas shown in white were not used in the comparison. Structures are colored by Procrustes score of the central residue of an aligned fragment pair according to the legend shown on the right. The following comparisons were made: AncGR2 LBD-TA-SHP versus AncGR2 LBD-Dex-TIF2 (PDB 3GN8); AncGR2 LBD-TA-SHP versus AncGR2 LBD-MF-TIF2 (PDB 4E2J); AncGR2 LBD-Dex-TIF2 versus AncGR2 LBD-MF-TIF2. (b) Structural overlay of the mouth of the ligand-binding pocket, which was one of the areas with the largest variance from the analysis in (a). TA induces a narrowing of the pocket entrance, indicated by a 4.6 A distance between Ser25 on H3 and Pro106 on H6. Dex has a 6.3-Å and MF has a 10.1-Å distance across the same area. (c) Five-hundred-nanosecond molecular dynamics simulations looking at root mean square fluctuations between various GR-ligand complexes. **Areas of significant differences in root mean square fluctuations that are not obvious due to flexible loops, where higher root mean square fluctuation values are to be expected. (d) The AncGR2 LBD-TA structure shows the acetonide group on TA makes hydrophobic contacts (black) with Met29 and Met108. (e) The hydrophobic contacts seen in (d) cause a shift in the position of other side chains within the ligand-binding pocket, such as Met103 (f). Structural overlays of AncGR2-ligand complexes show this is unique to the TA-bound complex. (g) The shift in side chains causes an overall rearrangement of $\mathrm{H} 6 / \mathrm{H} 7$, explaining the constricted pocket entrance seen in the TA structure.

peptide helix, which is further stabilized between the aromatic face and the Ser26 side chain. The NR box 1 peptide contains an arginine residue at the first position, but the density in this side chain was not strong enough to model; therefore, it is modeled as an alanine residue. The SHP NR box 1 and 2 peptides look most similar to the LXXLL motif seen in the coactivator PGC-1 $\alpha$ (Fig. $6 \mathrm{D})$. It has been postulated that SHP antagonizes PGC- $1 \alpha$ activation of GR. This repression of GR was shown to inhibit phosphoenolpyruvate carboxykinase, implying a role for SHP in modulating GR function within the liver (Borgius et al., 2002). 
A

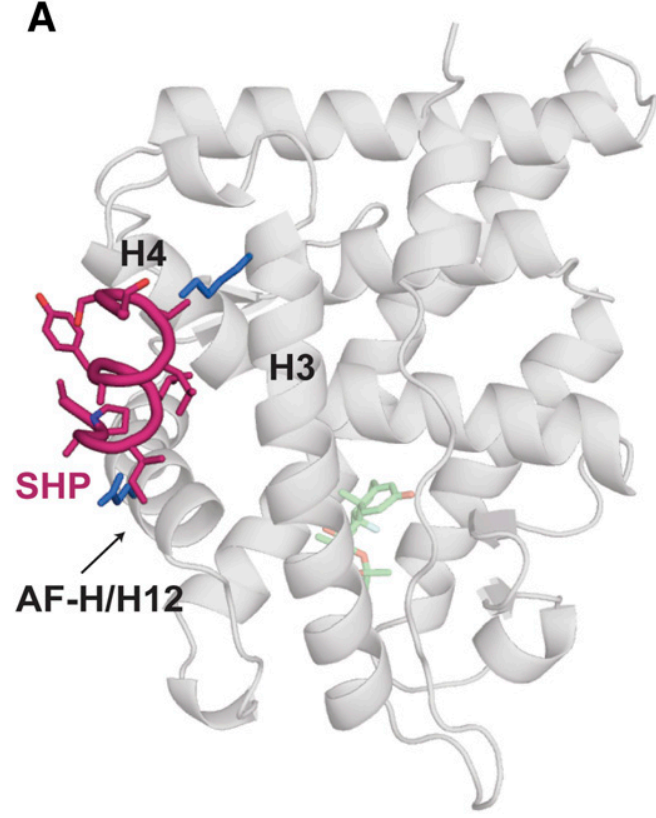

D ShP NR BOX 1 APAILYALLSS

SHP NR BOX 2 VPSILKKILLE

PGC-1a EPSLLKKLLLA

TIF-2 NR BOX 3 NALLRYLLDKD

LXXLL Motif
B

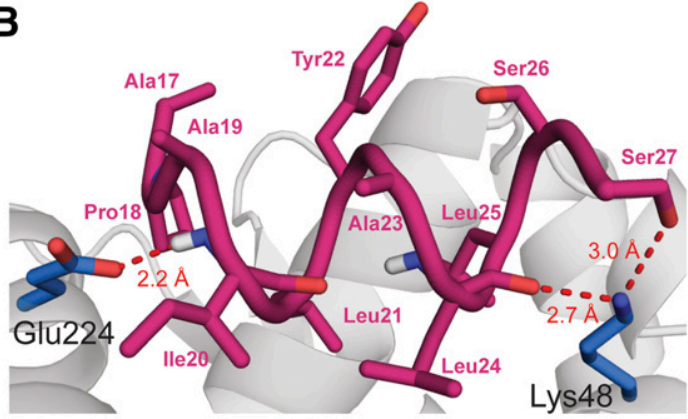

C
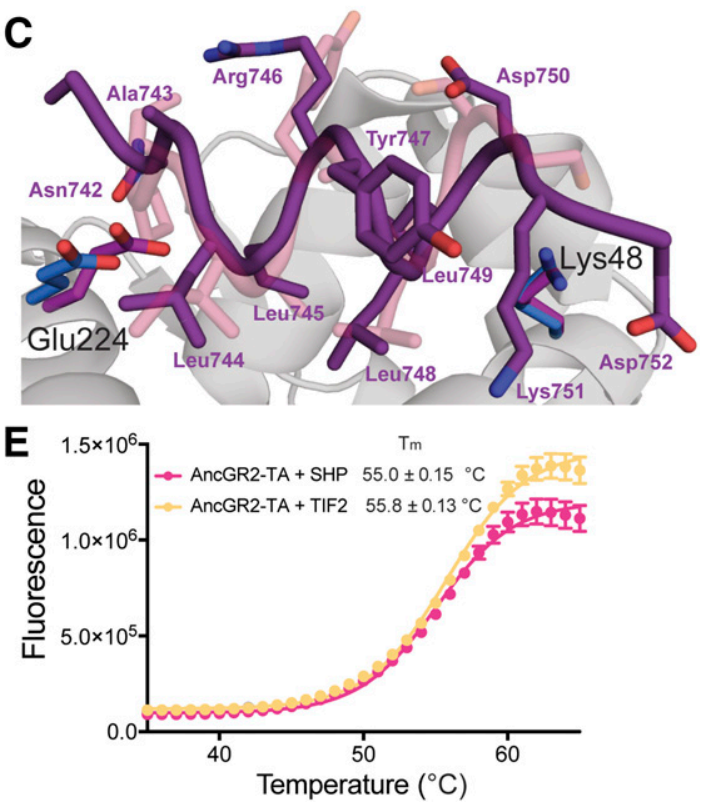

Fig. 6. AncGR2-TA complex is bound by atypical coregulator, SHP. (a) Overall structure of AncGR2 with SHP represented as sticks and ribbon (pink). The peptide is bound in the canonical AF-2 surface made by $\mathrm{H} 3, \mathrm{H} 4$, and AF-H/H12. The peptide is held in place by charge clamp formed by Glu224 and Lys48, shown in sticks (blue). (b) Zoom in of SHP peptide. (c) Overlay of SHP (faded pink) with TIF2 peptide (purple) from the AncGR2-Dex (PDB 3GN8) structure. (d) Comparison of LXXLL motifs found in various coregulators. (e) Differential scanning fluorimetry monitors the thermal stability of AncGR2 LBD-TA in complex with the SHP NR box 1 and TIF-2 NR box 3 peptide. There is little difference between the two complexes.

The SHP peptide in our structure interacts with the same AF-2 surface seen in the previously solved AncGR2 LBD-TIF2 NR box 3 structure (Fig. 6C) (Kohn et al., 2012). Since SHP interacts with GR via a classic NR coactivator motif, similar interactions are expected. In both GR-peptide complexes, the charge clamp formed by Lys48 and Glu224 is conserved. Despite these peptides having sequence differences, both peptides stabilize AncGR2 LBD to similar levels (Fig. 6, D and E).

\section{Discussion}

In the 1940s, the GR's endogenous ligand, cortisol, was identified as a potent suppressor of inflammation (Kendall, 1951). Since then, synthetic GCs with dramatically improved affinity, potency, and selectivity have become the most widely used treatment of anti-inflammatory therapies (Clark and Belvisi, 2012). Targeting GR is not challenging; however, current GCs drive the activation of metabolic, homeostatic, and growth pathways in addition to immunosuppression (Cato and Wade, 1996; De Bosscher et al., 2000; Schäcke et al., 2002). X-ray crystal structures are required to build robust structure-function relationships; however, this has proved difficult due to the instability of recombinantly expressed, purified hGR LBD. To circumvent this issue, we used the AncGR2 LBD derived from the phylogenetically reconstructed GR present in the ancestor of bony vertebrates (Bridgham et al., 2009; Kohn et al., 2012; Colucci and Ortlund, 2013). This ancestral receptor has been extensively used to understand the evolution of corticoid selectivity in modern GR; it displays the same ligand selectivity and agonist response as the human receptor but shows enhanced expression, solubility, crystallizability, and tolerance to mutation (Ortlund et al., 2007; Bridgham et al., 2009; Kohn et al., 2012). Therefore, AncGR2 represents a powerful tool to explore novel GR-ligand complexes that would otherwise be difficult to probe (Bridgham et al., 2009; Kohn et al., 2012; Colucci and Ortlund, 2013).

The synthetic glucocorticoid TA is used in the clinic to treat allergic rhinitis, macular edema, and skin lesions (Veritti et al., 2012; Fredman and Tenenhaus, 2013). TA is more hydrophobic than other synthetic GCs, making it ideal for topical use and allowing for a prolonged duration of action (Doggrell, 2001; Fredman and Tenenhaus, 2013). Furthermore, TA has gained popularity in the laboratory due to its increased affinity for GR over other synthetic compounds (Fig. 3C), such as dexamethasone (Yoneda et al., 1995; Veritti et al., 2012), and for its ability to promote the expression and solubility of the intact receptor. These improvements in 
affinity, potency, and bioavailability over other GCs make TA a critical ligand to study. We show that TA significantly increases the thermal stability of the LBD relative to dexamethasone (Fig. $3 \mathrm{D}$ ), causing GR to assume a more compact structure with smaller conformational fluctuations near the pocket (Fig. 5). Driving these effects with TA is the bulky hydrophobic C-17 acetonide moiety, which generates additional contacts relative to other ligands and repositions the H6-H7 loop to constrict the pocket (Fig. 5, D and E). The bound TA maintains interactions with the hydrogen-bond network critical for GR specificity and transactivation (Figs. 1D, 2, and 4A) but enables greater intramolecular contacts, which likely explains the increased affinity and stability (Fig. 5). Ligand-driven perturbation of intramolecular contacts and dynamics at the mouth of the ligand-binding pocket has been shown to be essential for selective modulation of other nuclear receptors, such as estrogen receptor $\mathrm{ER} \alpha$, peroxisome proliferator activated receptor $\operatorname{PPAR} \gamma$, and LRH1 (Gee and Katzenellenbogen, 2001; Musille et al., 2012; Kojetin and Burris, 2013; Srinivasan et al., 2013). Taken together, we have elucidated the structural mechanisms driving TA's enhanced affinity and ability to stabilize the GR LBD, which will inform future glucocorticoid design.

This work also offers the first visualization of GR's interaction with the atypical NR, SHP, which is part of a transcriptional corepressor complex that targets NRs in the active conformation (Båvner et al., 2002; Borgius et al., 2002; Macchiarulo et al., 2006; Zhi et al., 2014). SHP accomplishes this by utilizing two LXXLL motifs to mimic coactivators and bind directly to the AF surface. SHP is expressed at high levels in the liver and has been shown to interact directly with GR to modulate its function (Borgius et al., 2002). We demonstrate that, indeed, SHP binds to the AF surface on GR and makes the conserved charge clamp interaction, similar to the previously solved structure of AncGR2 in complex with the coregulator TIF2 (Fig. 6B). Furthermore, the GR LBD-TA complex was stabilized to similar levels whether in complex with SHP or TIF2 (Fig. 6E). SHP has been suggested to play a role in modulating hepatic GR function and, thereby, metabolism (Borgius et al., 2002). It is likely that increased levels of SHP permit direct competition with coregulators for the GR-agonist-DNA complex (Båvner et al., 2002; Borgius et al., 2002; Macchiarulo et al., 2006; Zhi et al., 2014). However, further studies are needed to test this mechanism and to determine its physiologic and clinical relevance for hepatic GR action.

Developing improved GCs with fewer off-target side effects will require linking ligand-induced receptor motions with selective coregulator interactions to drive GR to specific promoters (Nehmé et al., 2009; Sacta et al., 2016; Weikum et al., 2017). Obtaining structural and dynamic information, as presented here, is vital to this effort.

\section{Acknowledgments}

X-ray data were collected at Southeast Regional Collaborative Access Team 22-ID beamline at the Advanced Photon Source, Argonne National Laboratory. Supporting institutions may be found at www. ser.aps.anl.gov. Use of the Advanced Photon Source was supported by the U.S. Department of Energy, Office of Science, Office of Basic Energy Sciences, under contract W-31-109-Eng-38.

\section{Authorship Contributions}

Participated in research design: Weikum, Okafor, Ortlund.
Conducted experiments: Weikum, Okafor, D'Agostino, Ortlund. Performed data analysis: Weikum, Okafor, D’Agostino, Ortlund. Wrote or contributed to the writing of the manuscript: Weikum, Okafor, D'Agostino, Colucci, Ortlund.

\section{References}

Adams PD, Afonine PV, Bunkóczi G, Chen VB, Davis IW, Echols N, Headd JJ, Hung LW, Kapral GJ, Grosse-Kunstleve RW, et al. (2010) PHENIX: a comprehensive Python-based system for macromolecular structure solution. Acta Crystallogr D Biol Crystallogr 66:213-221.

Bain DL, Yang Q, Connaghan KD, Robblee JP, Miura MT, Degala GD, Lambert JR, and Maluf NK (2012) Glucocorticoid receptor-DNA interactions: binding energetics are the primary determinant of sequence-specific transcriptional activity. $J$ Mol Biol 422:18-32.

Båvner A, Johansson L, Toresson G, Gustafsson JA, and Treuter E (2002) A transcriptional inhibitor targeted by the atypical orphan nuclear receptor SHP. EMBO Rep 3:478-484

Bikowski J, Pillai R, and Shroot B (2006) The position not the presence of the halogen in corticosteroids influences potency and side effects. J Drugs Dermatol 5:125-130.

Bledsoe RK, Montana VG, Stanley TB, Delves CJ, Apolito CJ, McKee DD, Consler TG, Parks DJ, Stewart EL, Willson TM, et al. (2002) Crystal structure of the glucocorticoid receptor ligand binding domain reveals a novel mode of receptor dimerization and coactivator recognition. Cell 110:93-105.

Borgius LJ, Steffensen KR, Gustafsson JA, and Treuter E (2002) Glucocorticoid signaling is perturbed by the atypical orphan receptor and corepressor SHP. J Biol Chem 277:49761-49766.

Bridgham JT, Ortlund EA, and Thornton JW (2009) An epistatic ratchet constrains the direction of glucocorticoid receptor evolution. Nature 461:515-519.

Carson-Jurica MA, Schrader WT, and O'Malley BW (1990) Steroid receptor family: structure and functions. Endocr Rev 11:201-220.

Case D, Babin V, Berryman J, Betz R, Cai Q, Cerutti D, Cheatham Iii T, Darden T, Duke R and Gohlke H (2014) Amber 14. University of California, San Francisco.

Case D and Kollman P (2012) AmberTools 12. University of California, San Francisco.

Cato AC and Wade E (1996) Molecular mechanisms of anti-inflammatory action of glucocorticoids. BioEssays 18:371-378.

Clark AR and Belvisi MG (2012) Maps and legends: the quest for dissociated ligands of the glucocorticoid receptor. Pharmacol Ther 134:54-67.

Colucci JK and Ortlund EA (2013) X-ray crystal structure of the ancestral 3-ketosteroid receptor-progesterone-mifepristone complex shows mifepristone bound at the coactivator binding interface. PLoS One 8:e80761.

Darimont BD, Wagner RL, Apriletti JW, Stallcup MR, Kushner PJ, Baxter JD, Fletterick RJ, and Yamamoto KR (1998) Structure and specificity of nuclear receptor-coactivator interactions. Genes Dev 12:3343-3356.

Davis IW, Leaver-Fay A, Chen VB, Block JN, Kapral GJ, Wang X, Murray LW, Arendall WB, 3rd, Snoeyink J, and Richardson JS et al. (2007) MolProbity: allatom contacts and structure validation for proteins and nucleic acids. Nucleic Acids Res 35:W375-W383.

De Bosscher K, Vanden Berghe W, and Haegeman G (2000) Mechanisms of antiinflammatory action and of immunosuppression by glucocorticoids: negative interference of activated glucocorticoid receptor with transcription factors. $J$ Neuroimmunol 109:16-22

Derendorf H and Meltzer EO (2008) Molecular and clinical pharmacology of intranasal corticosteroids: clinical and therapeutic implications. Allergy 63: $1292-1300$

Doggrell SA (2001) Triamcinolone: new and old indications. Expert Opin Pharmacother 2:1177-1186

Emsley P, Lohkamp B, Scott WG, and Cowtan K (2010) Features and development of Coot. Acta Crystallogr D Biol Crystallogr 66:486-501.

Feig M, Karanicolas J, and Brooks, 3rdCL (2004) MMTSB Tool Set: enhanced sampling and multiscale modeling methods for applications in structural biology. $J$ Mol Graph Model 22:377-395.

Fredman R and Tenenhaus M (2013) Cushing's syndrome after intralesional triamcinolone acetonide: a systematic review of the literature and multinational survey. Burns 39:549-557.

Gebhardt JC, Suter DM, Roy R, Zhao ZW, Chapman AR, Basu S, Maniatis T, and Xie XS (2013) Single-molecule imaging of transcription factor binding to DNA in live mammalian cells. Nat Methods 10:421-426.

Gee AC and Katzenellenbogen JA (2001) Probing conformational changes in the estrogen receptor: evidence for a partially unfolded intermediate facilitating ligand binding and release. Mol Endocrinol 15:421-428.

Gronemeyer H and Moras D (1995) Nuclear receptors. How to finger DNA. Nature 375:190-191.

Grossmann C, Scholz T, Rochel M, Bumke-Vogt C, Oelkers W, Pfeiffer AF, Diederich $\mathrm{S}$, and Bahr V (2004) Transactivation via the human glucocorticoid and mineralocorticoid receptor by therapeutically used steroids in CV-1 cells: a comparison of their glucocorticoid and mineralocorticoid properties. Eur J Endocrinol 151: $397-406$.

Heery DM, Kalkhoven E, Hoare S, and Parker MG (1997) A signature motif in transcriptional co-activators mediates binding to nuclear receptors. Nature 387:733-736. Hu X and Lazar MA (1999) The CoRNR motif controls the recruitment of corepressors by nuclear hormone receptors. Nature 402:93-96.

Hudson WH, Youn C, and Ortlund EA (2013) The structural basis of direct glucocorticoid-mediated transrepression. Nat Struct Mol Biol 20:53-58.

Jenkins BD, Pullen CB, and Darimont BD (2001) Novel glucocorticoid receptor coactivator effector mechanisms. Trends Endocrinol Metab 12:122-126.

Johansson L, Båvner A, Thomsen JS, Färnegårdh M, Gustafsson JA, and Treuter E (2000) The orphan nuclear receptor SHP utilizes conserved LXXLL-related motifs 
for interactions with ligand-activated estrogen receptors. Mol Cell Biol 20: 1124-1133.

Johansson L, Thomsen JS, Damdimopoulos AE, Spyrou G, Gustafsson JA and Treuter E (1999) The orphan nuclear receptor SHP inhibits agonist-dependent transcriptional activity of estrogen receptors ERalpha and ERbeta. J Biol Chem 274:345-353.

Joosten RP, Salzemann J, Bloch V, Stockinger H, Berglund AC, Blanchet C, BongcamRudloff E, Combet C, Da Costa AL, Deleage G, et al. (2009) PDB_REDO: automated re-refinement of X-ray structure models in the PDB. J Appl Cryst 42: 376-384.

Jorgensen WL, Chandrasekhar J, Madura JD, Impey RW, and Klein ML (1983) Comparison of simple potential functions for simulating liquid water. J Chem Phys 79:926-935.

Kadmiel M and Cidlowski JA (2013) Glucocorticoid receptor signaling in health and disease. Trends Pharmacol Sci 34:518-530.

Kendall EC (1951) The development of cortisone as a therapeutic agent. Antibiot Chemother (Northfield) 1:7-15.

Kohn JA, Deshpande K, and Ortlund EA (2012) Deciphering modern glucocorticoid cross-pharmacology using ancestral corticosteroid receptors. J Biol Chem 287: 16267-16275.

Kojetin DJ and Burris TP (2013) Small molecule modulation of nuclear receptor conformational dynamics: implications for function and drug discovery. Mol Pharmacol 83:1-8.

Kumar R and Thompson EB (1999) The structure of the nuclear hormone receptors. Steroids 64:310-319.

Lee YK, Dell H, Dowhan DH, Hadzopoulou-Cladaras M, and Moore DD (2000) The orphan nuclear receptor SHP inhibits hepatocyte nuclear factor 4 and retinoid X receptor transactivation: two mechanisms for repression. Mol Cell Biol 20:187-195.

Lee YS, Chanda D, Sim J, Park YY, and Choi HS (2007) Structure and function of the atypical orphan nuclear receptor small heterodimer partner. Int Rev Cytol 261:117-158.

Luisi BF, Xu WX, Otwinowski Z, Freedman LP, Yamamoto KR, and Sigler PB (1991) Crystallographic analysis of the interaction of the glucocorticoid receptor with DNA. Nature 352:497-505.

Macchiarulo A, Rizzo G, Costantino G, Fiorucci S, and Pellicciari R (2006) Unveiling hidden features of orphan nuclear receptors: the case of the small heterodimer partner (SHP). J Mol Graph Model 24:362-372.

McDonald IK and Thornton JM (1994) Satisfying hydrogen bonding potential in proteins. J Mol Biol 238:777-793.

Millard CJ, Watson PJ, Fairall L, and Schwabe JW (2013) An evolving understanding of nuclear receptor coregulator proteins. J Mol Endocrinol 51:T23-T36.

Mordacq JC and Linzer DI (1989) Co-localization of elements required for phorbol ester stimulation and glucocorticoid repression of proliferin gene expression. Genes Dev 3:760-769.

Musille PM, Pathak MC, Lauer JL, Hudson WH, Griffin PR, and Ortlund EA (2012) Antidiabetic phospholipid-nuclear receptor complex reveals the mechanism for phospholipid-driven gene regulation. Nat Struct Mol Biol 19:532-537.

Nagy L and Schwabe JW (2004) Mechanism of the nuclear receptor molecular switch. Trends Biochem Sci 29:317-324.

Nehmé A, Lobenhofer EK, Stamer WD, and Edelman JL (2009) Glucocorticoids with different chemical structures but similar glucocorticoid receptor potency regulate subsets of common and unique genes in human trabecular meshwork cells. BMC Med Genomics 2:58.

Nicholls RA, Fischer M, McNicholas S, and Murshudov GN (2014) Conformationindependent structural comparison of macromolecules with ProSMART. Acta Crystallogr D Biol Crystallogr 70:2487-2499.

O'Malley BW and Tsai MJ (1992) Molecular pathways of steroid receptor action. Biol Reprod 46:163-167.

Ortlund EA, Bridgham JT, Redinbo MR, and Thornton JW (2007) Crystal structure of an ancient protein: evolution by conformational epistasis. Science 317:1544-1548
Ortlund EA, Lee Y, Solomon IH, Hager JM, Safi R, Choi Y, Guan Z, Tripathy A, Raetz CR, McDonnell DP, et al. (2005) Modulation of human nuclear receptor LRH-1 activity by phospholipids and SHP. Nat Struct Mol Biol 12:357-363.

Otwinowski Z and Minor W (1997) Processing of X-ray diffraction data collected in oscillation mode. Methods Enzymol 276:307-326.

Pérez A, Marchán I, Svozil D, Sponer J, Cheatham, 3rdTE, Laughton CA, and Orozco M (2007) Refinement of the AMBER force field for nucleic acids: improving the description of $\alpha / \gamma$ conformers. Biophys $J$ 92:3817-3829.

Pratt WB and Toft DO (1997) Steroid receptor interactions with heat shock protein and immunophilin chaperones. Endocr Rev 18:306-360.

Robblee JP, Miura MT, and Bain DL (2012) Glucocorticoid receptor-promoter interactions: energetic dissection suggests a framework for the specificity of steroid receptor-mediated gene regulation. Biochemistry 51:4463-4472.

Roe DR and Cheatham, 3rdTE (2013) PTRAJ and CPPTRAJ: software for processing and analysis of molecular dynamics trajectory data. J Chem Theory Comput $\mathbf{9}$ 3084-3095.

Ryckaert J-P, Ciccotti G, and Berendsen HJ (1977) Numerical integration of the cartesian equations of motion of a system with constraints: molecular dynamics of n-alkanes. J Comput Phys 23:327-341.

Sacta MA, Chinenov Y, and Rogatsky I (2016) Glucocorticoid Signaling: An Update from a Genomic Perspective. Annu Rev Physiol 78:155-180.

Schäcke H, Döcke WD, and Asadullah K (2002) Mechanisms involved in the side effects of glucocorticoids. Pharmacol Ther 96:23-43.

Schüle R, Rangarajan P, Kliewer S, Ransone LJ, Bolado J, Yang N, Verma IM, and Evans RM (1990) Functional antagonism between oncoprotein c-Jun and the glucocorticoid receptor. Cell 62:1217-1226.

Seol W, Choi HS, and Moore DD (1996) An orphan nuclear hormone receptor that lacks a DNA binding domain and heterodimerizes with other receptors. Science 272:1336-1339.

Srinivasan S, Nwachukwu JC, Parent AA, Cavett V, Nowak J, Hughes TS, Kojetin DJ, Katzenellenbogen JA, and Nettles KW (2013) Ligand-binding dynamics rewire cellular signaling via estrogen receptor- $\alpha$. Nat Chem Biol 9:326-332.

Thiruchelvam PT, Lai CF, Hua H, Thomas RS, Hurtado A, Hudson W, Bayly AR, Kyle FJ, Periyasamy M, Photiou A, et al. (2011) The liver receptor homolog-1 regulates estrogen receptor expression in breast cancer cells. Breast Cancer Res Treat 127:385-396.

Vandevyver S, Dejager L, and Libert C (2014) Comprehensive overview of the structure and regulation of the glucocorticoid receptor. Endocr Rev 35:671-693.

Veritti D, Di Giulio A, Sarao V, and Lanzetta P (2012) Drug safety evaluation of intravitreal triamcinolone acetonide. Expert Opin Drug Saf 11:331-340.

Weikum ER, Knuesel MT, Ortlund EA, and Yamamoto KR (2017) Glucocorticoid receptor control of transcription: precision and plasticity via allostery. Nat Rev Mol Cell Biol 18:159-174.

Yang-Yen HF, Chambard JC, Sun YL, Smeal T, Schmidt TJ, Drouin J, and Karin M (1990) Transcriptional interference between c-Jun and the glucocorticoid receptor: mutual inhibition of DNA binding due to direct protein-protein interaction. Cell 62: $1205-1215$

Yoneda Y, Han D, Ogita K, and Watanabe A (1995) Distinction between binding of $[3 \mathrm{H}]$ triamcinolone acetonide to a ligand binding domain on the glucocorticoid receptor complex in cytosol fractions of brain and liver from the rat with intact adrenals. Brain Res 685:105-116.

Zhi X, Zhou XE, He Y, Zechner C, Suino-Powell KM, Kliewer SA, Melcher K, Mangelsdorf DJ, and Xu HE (2014) Structural insights into gene repression by the orphan nuclear receptor SHP. Proc Natl Acad Sci USA 111:839-844.

Address correspondence to: Eric A. Ortlund, 1510 Clifton Road NE, Rollins Research Center G235, Atlanta, GA 30322. E-mail: eortlun@emory.edu 